\title{
Is Radiocholine PET/CT Already Clinically Useful in Patients with Prostate Cancer?
}

$\mathbf{I}_{\mathrm{n}}$ (1) suggest the use of ${ }^{11} \mathrm{C}$-choline PET/CT before salvage radiation therapy during the early phase of biochemical relapse in patients with prostate cancer ( $\mathrm{PCa})$. Because of the high probability of detecting positive findings outside the pelvis due to rapidly increasing prostatespecific antigen (PSA) levels, both as absolute and as kinetic values, despite ongoing androgen deprivation therapy, ${ }^{11} \mathrm{C}$-choline may select patients who really benefit from salvage radiation therapy. But what is the current clinical role of radiolabeled choline in patients with PCa?

In the developed western countries, $\mathrm{PCa}$ is the second most common malignant neoplasm and the sixth cause of cancer-related death in men. The diagnosis is rarely done before age 50, becoming more frequent with advancing age. Usually PCa shows

\section{See page 1424}

a favorable prognosis, growing slowly and being well differentiated and frequently asymptomatic; as a consequence, many men affected with this neoplasm undergo no diagnosis or therapy, dying of other unrelated causes. Nevertheless, in patients undergoing surgery or curative radiotherapy (or brachytherapy) with or without androgen deprivation therapy, a high rate of recurrences occurs, appearing as local relapse or lymph node or bone/visceral metastases. Fewer cases are lethal, with lethal cases being mainly those in which castration-resistant secondary lesions are present. Although it increases cancer detection, a screening using PSA testing is not recommended because of its moderate sensitivity and specificity $(60 \%-80 \%)$ and thus high risk of overdiagnosis and overtreatment. Furthermore, mortality benefit from screening has not been demonstrated.

\section{DIAGNOSIS}

More extensive diagnostic testing may be individually suggested in men aged $50 \mathrm{y}$ and older, on the basis of symptoms and risk factors related to issues such as age, race, family history, and sexual habits. Symptoms, such as disorders in micturition or problems during sexual intercourse, may be similar to those caused by a benign disease. The diagnostic approach starts with a digital rectal examination and PSA testing. The only test that can fully confirm the diagnosis is a biopsy by transrectal ultrasonography, mandatory

Received Jun. 16, 2014; revision accepted Jun. 18, 2014

For correspondence or reprints contact: Luigi Mansi, Medicina Nucleare, Seconda Università di Napoli, Piazza Miraglia, 80138 Napoli, Italy.

E-mail: luigi.mansi@unina2.it

Published online Jul. 24, 2014.

COPYRIGHT (C 2014 by the Society of Nuclear Medicine and Molecular Imaging, Inc.

DOI: $10.2967 /$ jnumed.114.142679 for a prognostic evaluation, mainly based on histologic grading, called the Gleason score (GS). MR imaging may also allow an increasing accuracy in diagnosing PCa, mainly when functional techniques (functional MR imaging) or spectroscopy (MR spectroscopy) are feasible.

Staging is based on the TNM classification (2). Most important is the distinction between pathologically organ-defined PCa (pT12), showing a favorable prognosis, and nonconfined neoplasm (pT3-4), with substantially diminished chances of cure. The spread within the pelvis is evaluated by CT or MR imaging. Endorectal MR imaging allows the extension of disease to the prostatic capsule and seminal vesicles to be closely analyzed. MR imaging is preferable in the active surveillance of subjects with diagnosed PCa at low risk (i.e., confined PCa, GS $<6$ and PSA $<10 \mathrm{ng} / \mathrm{mL}$ ). The option to observe should always be made in terms of life expectancy and quality. In accordance with European $(3,4)$ and American guidelines (5), nuclear medicine has traditionally been limited to bone scanning (BS) in patients scheduled for primary treatment. Because of a more favorable cost and effectiveness (although improved accuracy is achievable with SPECT or SPECT/CT and even more so using PET/CT with ${ }^{18}$ F-fluoride [PET NaF]), BS remains the first-line standard choice for the early assessment of bone involvement in many institutions.

\section{RADIOCHOLINE AND RADIOACETATE IN PRETHERAPEUTIC EVALUATION}

Because of a low sensitivity, PET with ${ }^{18} \mathrm{~F}-\mathrm{FDG}$ is not indicated in $\mathrm{PCa}$, but in the case of suspicion of tumor dedifferentiation - that is, in patients with advanced disease or in subjects with a high GS or high PSA values or even in the presence of castration-resistant lesions-anaerobic glucidic metabolism seems more representative. To overcome ${ }^{18}$ F-FDG limitations, alternative radiotracers have been proposed and clinically evaluated. Choline is involved in phospholipid synthesis and integrity of cell membranes, particularly in the case of malignant proliferation; an increased uptake of radiocholine is observed in many neoplasms other than PCa (e.g., breast, liver, and brain tumors) in the presence of an aerobic metabolism or a low growing rate. Unfortunately, in patients with $\mathrm{PCa}$, results that are either false-negative-mainly in primary lesions under $5 \mathrm{~mm}$ or in minimally involved lymph nodes-or false-positive-due to benign prostatic lesions and, more rarely, to reactive lymph nodes-are frequent. Compared with ${ }^{18} \mathrm{~F}$-choline, ${ }^{11} \mathrm{C}$-choline allows a better evaluation of the pelvic region, because of a lower urinary excretion (although a greater bowel elimination). ${ }^{18} \mathrm{~F}$-choline has the advantage of being available in centers without a cyclotron and has evidenced a substantially global overlapping diagnostic accuracy with respect to ${ }^{11} \mathrm{C}$-choline, because of a slightly higher sensitivity for bone metastases.

Although radioacetate is likewise involved in the synthesis of cell membranes, there are fewer reports in the literature describing 
radioacetate, labeled with ${ }^{11} \mathrm{C}$ or ${ }^{18} \mathrm{~F}$, that show a similar pathophysiologic behavior with respect to radiocholine. The minor diffusion for radioacetate can be explained by less favorable technical issues and by a slightly lower uptake in aerobic conditions, frequently prevalent in $\mathrm{PCa}$. Because the number of false-negative and false-positive results in the pretherapeutic phase is high for both radiocholine and radioacetate, neither is currently indicated in the diagnosis or staging of PCa patients; rather, these tracers are used only in certain individual cases. Similarly, radiocholine and radioacetate are not suggested to define a target in radiotherapy because of the high number of false-negative lymph nodes.

\section{RESTAGING}

Radiocholine and radioacetate have been proposed in the early restaging of patients in whom local or distant recurrences are suggested. After surgery or local radiation therapy, PSA values and kinetics are usually monitored. In the case of an increase in PSA levels $(>0.2 \mathrm{ng} / \mathrm{mL}$ and $>$ PSA nadir, respectively, after surgery and radiotherapy), MR imaging or BS is advised although these are not always effective for an early and reliable detection of recurrence. In this clinical setting, radiocholine is indicated to individuate prostatic fossae, local/ distant lymph nodes, and bone/visceral metastases, assuming a direct influence on deciding therapeutic strategies or in helping to avoid unnecessary therapies. For the detection of prostatic fossae recurrence, the urinary excretion and accumulation in the bowel, respectively, for ${ }^{18} \mathrm{~F}$-choline and ${ }^{11} \mathrm{C}$ choline can reduce the sensitivity of the imaging modality. Moreover, radiocholine PET is not yet considered a perfect substitute for osteotropic agents, because of the possible presence of osteoblastic lesions visible only by NaF PET and not by radiocholine. Furthermore, although bone marrow metastases undetectable by NaF PET may be found with radiocholine, BS (or NaF PET) remains indispensable to recruit patients undergoing palliative $\left({ }^{153} \mathrm{Sm}\right.$ or $\left.{ }^{89} \mathrm{Sr}\right)$ or curative $\left(\alpha\right.$-emitter $\left.{ }^{223} \mathrm{Ra}\right)$ radionuclide therapy for diffuse skeletal metastases. Many studies have clearly demonstrated the impact of radiocholine PET/CT on the change in treatment management, through the detection or exclusion of local recurrence or the finding of unexpected distant metastases. Many reports have tried to individuate a reliable PSA threshold for increasing the performance of radiocholine PET, thus reducing the rate of false-negative and false-positive results. A strategy redefining PSA kinetics and trigger PSA together would be advisable. In fact, some authors recommend radiocholine PET in patients with a biochemical relapse of disease in the case of PSA greater than $1.5 \mathrm{ng} / \mathrm{mL}$, PSA velocity greater than $0.75 \mathrm{ng} / \mathrm{mL} / \mathrm{y}$, or PSA doubling time less than 6 mo.

\section{PET/MR IMAGING}

A certain improvement for radiocholine will come from PET/MR imaging, which allows an increased diagnostic accuracy with respect to PET/CT, because of a more precise analysis of pelvic lesions, favored by the superiority of MR imaging in evaluating soft tissues. Promising perspectives could be realized, probably also in the pretherapeutic phase, through the implemental contribution of functional MR imaging and MR spectroscopy. Interestingly, an increased sensitivity in individuating skeletal metastases could be provided by PET/MR imaging with $\mathrm{NaF}$, allowing an early detection either of osteoblastic lesions (with $\mathrm{NaF}$ ) or of metastases confined in the bone marrow (with MR imaging).

\section{AVAILABLE AND NEWER RADIOTRACERS}

In patients who are candidates for radiotherapy, either primary or salvage treatment, radiotracers evaluating hypoxia such as ${ }^{18} \mathrm{~F}$-misonidazole or ${ }^{18} \mathrm{~F}$-fluoroazomycin arabinoside could be useful, permitting a better biologic definition of the tumor target. In this context, a PET scan with $3^{\prime}$-deoxy$3^{\prime}-{ }^{18}$ F-fluorothymidine could be suggested to define a field of irradiation, reducing, as much as possible, side effects due to the radiation therapy in the surrounding tissues, particularly in bone marrow in the pelvis. Interestingly, $3^{\prime}$-deoxy-3'-18 Ffluorothymidine could be used also for evaluating the metabolic response to chemotherapy in patients with advanced disease. Conversely, for the assessment of the feasibility of androgen deprivation therapy in patients with skeletal metastases, there is great interest in the use of ${ }^{18} \mathrm{~F}$-fluoro-5a-dihydrotestosterone to target androgen receptors.

The most attractive newer radiotracers are those that target markers of the neoplastic prostatic cell, such as the prostatespecific membrane antigen (PSMA). The ligands with PSMA have already been labeled with many radionuclides, such as ${ }^{89} \mathrm{Zr},{ }^{64} \mathrm{Cu}$, ${ }^{11} \mathrm{C},{ }^{18} \mathrm{~F},{ }^{86} \mathrm{Y},{ }^{90} \mathrm{Y},{ }^{177} \mathrm{Lu}$, and ${ }^{68} \mathrm{Ga}$. In particular, ${ }^{68} \mathrm{Ga}$-PSMA has shown significant advantages in visualizing $\mathrm{PCa}$, differentiating inflammation or benign lesions from malignant ones, and better detecting lymph node and bone metastases than radiocholine PET. The availability of PSMA ligands labeled with ${ }^{177} \mathrm{Lu}$ or ${ }^{90} \mathrm{Y}$ supports a theranostic strategy with curative effects also in castration-resistant patients with diffuse bone metastases. Concerning theranostic models, similar results could also be achieved using ${ }^{64} \mathrm{Cu}$ or radioiodinated tracers. Monoclonal antibodies (i.e., J591, labeled either with radioiodine or with ${ }^{177} \mathrm{Lu}$ ) and radiolabeled ${ }^{64} \mathrm{Cu}$ molecules (e.g., ${ }^{64} \mathrm{Cu}-\mathrm{Cl} 2$ and ${ }^{64} \mathrm{Cu}$-DOTAAE105), usable either for PET or for radionuclide therapy, have already been evaluated in preclinical studies. Moreover, synthetic L-leucine analogs (e.g., ${ }^{18}$ F-FACBC [1-amino-3-fluorocyclobutane-1carboxylic acid] or ${ }^{18} \mathrm{~F}$-flucicovine) tracing amino-acid transport, ${ }^{68} \mathrm{Ga}$ bombesin analogs that bind to the human gastrinreleasing peptide receptor, antibody fragments directed to prostate stem cell antigen ( ${ }^{124} \mathrm{I}-\mathrm{A} 11$ PSCA), thymidine kinase radiotracers for assessing cellular proliferation and cellular stress, reporter probes for direct visualization and characterization of sentinel nodes in $\mathrm{PCa}$, radioligands for urotensin receptors that are involved in human prostate tumorigenesis, and radiolabeled new experimental drugs (e.g., ${ }^{124} \mathrm{I}-\mathrm{PUH} 71$ ) are now under investigation.

In conclusion, radiocholine PET may be considered a useful tool in the restaging of $\mathrm{PCa}$ patients. A certain diagnostic improvement would be achieved with PET/MR imaging, and further studies are needed both to evaluate the best strategy in the early detection of bone metastases and to include in the clinical setting the new radiopharmaceutical agents. A promising future is expected by nuclear medicine physicians and scientists thanks to the contribution both in the earlier diagnosis of recurrences and in the stratification of patients who are candidates for welldefined therapies. Moreover, the promise of improved prognosis in patients with advanced disease or castration-resistant cancer is awaited. An intriguing approach already in use in the clinical arena uses a radiotracer directed to a marker of the neoplastic prostatic cell-that is, PSMA labeled with either positron or $\beta$ emitters. 


\section{DISCLOSURE}

No potential conflict of interest relevant to this article was reported.

Luigi Mansi Seconda Università di Napoli Napoli, Italy

Vincenzo Cuccurullo Seconda Università di Napoli Napoli, Italy

Laura Evangelista Istituto Oncologico Veneto (IOV-IRCCS) Padova, Italy

\section{REFERENCES}

1. Castellucci P, Ceci F, Graziani T, et al. Early biochemical relapse after radical prostatectomy: which prostate cancer patient may benefit from a restaging ${ }^{11} \mathrm{C}$ choline PET/CT scan before salvage radiation therapy? J Nucl Med. 2014;55: $1424-1429$.

2. Edge SB, Byrd DR, Compton CC, et al., eds. AJCC Cancer Staging Manual. 7th ed. New York, NY: Springer; 2010:457-468.

3. Heidenreich A, Bastian PJ, Bellmunt J, et al. European Association of Urology. EAU guidelines on prostate cancer. Part 1: screening, diagnosis, and local treatment with curative intent-update 2013. Eur Urol. 2014;65:124-137.

4. Heidenreich A, Bastian PJ, Bellmunt J, et al. EAU guidelines on prostate cancer. Part II: treatment of advanced, relapsing, and castration-resistant prostate cancer. Eur Urol. 2014;65:467-479.

5. Mohler JL, Kantoff PW, Armstrong AJ, et al. Prostate cancer, version 2.2014. J Natl Compr Canc Netw. 2014;12:686-718. 